\title{
Characterization and Modeling of Growth and Remodeling in Tendon and Soft Tissue Constructs
}

\author{
E. M. Arruda ${ }^{1,2}$, S. C. Calve $^{2}$, K. Garikipati ${ }^{1}$, K. Grosh ${ }^{1,3}$, H. Narayanan ${ }^{1}$ \\ 1 Department of Mechanical Engineering \\ 2 Macromolecular Science and Engineering Program \\ 3 Department of Biomedical Engineering \\ University of Michigan, 2250 GG Brown, Ann Arbor, MI 48109, USA arruda@umich.edu, \\ scalve@umich.edu, krishna@umich.edu, grosh@umich.edu, hnarayan@umich.edu
}

Engineered tendon fibroblast and collagen-based soft tissue constructs have been developed to characterize growth and remodeling in soft tissue via in-vitro studies of the effects of controlled mechanical interventions on collagen content and orientation. Growth and remodeling often occur simultaneously in soft tissue. The present contribution distinguishes these separate biological processes and develops the theoretical frameworks for the formation of constitutive theories of each. Remodeling is considered a motion in material space that occurs at constant mass whereas growth is a change in concentration due to mass transport and is treated via a system open with respect to mass. Examples illustrating both biological processes are presented.

\section{Introduction}

Understanding growth and remodeling processes in tendon in health and disease is of great interest because of the role of tendon in transmitting forces produced by muscles during movement. Tendon is also an excellent testbed for studying collagen growth and remodeling processes in soft tissue because it is relatively acellular and avascular. Mature tendon consists largely of type I collagen. Therefore the mechanical properties of mature tendon can be largely attributed to the parallel collagen fibrils that comprise approximately $75 \%$ [Nordin (2001)] of its dry weight. Growth and remodeling experiments on soft tissue are challenging for many reasons as the data in Figure 1 demonstrate [Arruda et al. (2005)]. The normal tibialis anterior (TA) tendon of a rat is functionally graded along its length. In addition to measuring mechanical properties locally (achieved here via local optical strain measurement), assays for collagen content, cross-linking and cell expression must all be applied locally to formulate relationships between the biochemical variables and the mechanical properties. Knowledge of in vivo mechanical loading histories in tendon is very difficult to obtain and under much less control. Yet it is clear that mechanical loading is critical to collagen growth and remodeling as can be seen for example in Figure 1 in which a TA tendon that has been unloaded in vivo for a period 


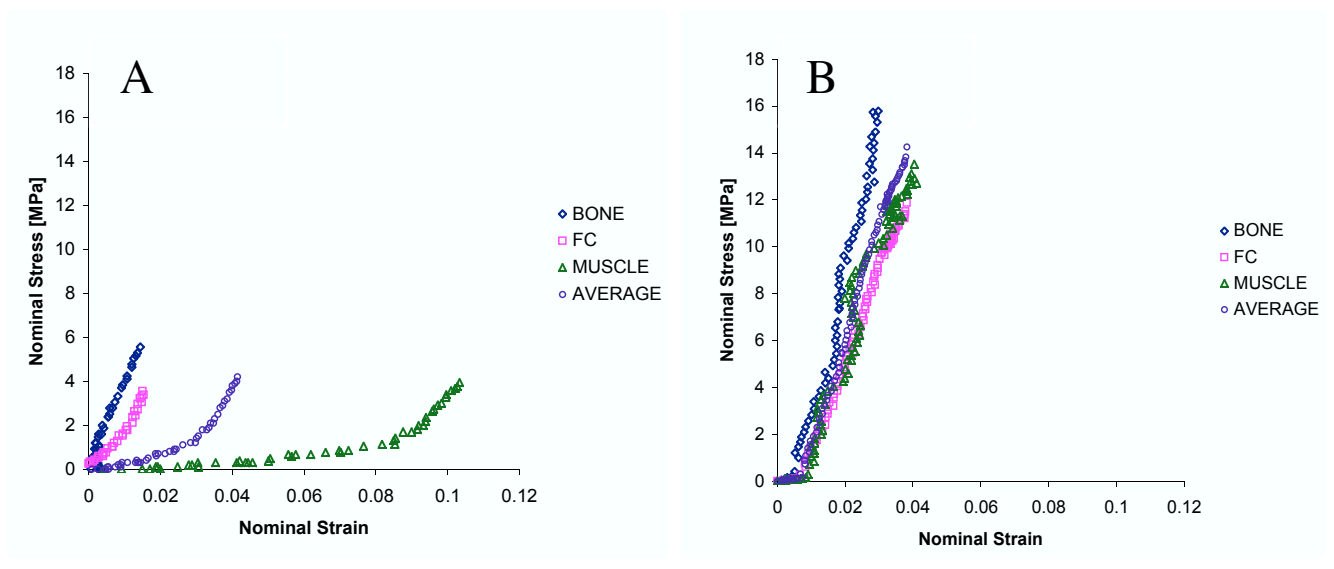

Figure 1. Mechanical response curve of the mature TA tendon of the rat at three local regions along the length of the tendon: BONE, distal end (near the bone); FC, fibrocartilage midsection; and MUSCLE, proximal end (near the muscle). The average response AVERAGE along the length is also shown. A Normal tendon is functionally graded, B After five weeks of unloading the previously compliant tendon regions have stiffened and the response is very uniform along its length.

of time is no longer functionally graded; regions that were relatively compliant under normal loading have undergone significant growth or remodeling to stiffen [Arruda et al. (2005)]. The so-called toe-region or initially compliant non-linear portion of the normal tendon is truncated as a result of the unloading and the tendon prematurely stiffens with increased tangent stiffness.

To conduct controlled experiments of the effects of mechanical loading on collagen growth and remodeling we have developed in-vitro models of soft tissue constructs comprised largely of type I collagen and tendon fibroblasts (tenocytes), and functional bioreactors for the application of cyclic mechanical interventions. The tissue engineering methods are described in Section 2 along with preliminary data demonstrating the growth response of the cells in these constructs subjected to static and cyclic mechanical loading protocols. Continuum level mathematical models of growth in soft tissue are currently a very active area of research [Epstein and Maugin (2000); Klisch and van Dyke (2001); Taber and Humphrey (2001); Humphrey and Rajagopal (2002); Kuhl and Steinmann (2002); Sengers et al. (2004)]. These models all consider the addition of mass as at least a subset of the phenomenon of growth. Some consider a single species (e.g. collagen) and consider its increasing mass. Others specifically include the transport of the growing species in a system that is open with respect to mass. Some of these formulations also describe a change in structural organization as a growth response. The present formulation, described in Sections 3 and 4, distinguishes between changes in material configurations at constant mass and growth processes. The former, described in Section 3, is treated as a motion in material space and is termed remodeling. The growth treatment in Section 4 describes transport of species such as nutrients and enzymes involved in tissue growth 
along with the tissues fluid species, and not the mass transport of the growing extracellular collagen matrix itself. This is in contrast to the previous treatments in which the growing species (e.g. collagen) diffuses, but it is consistent with many physiologically relevant homeostatic tissue growth phenomena found in soft tissue growth.

\section{Engineered Tissue Constructs}

For growth and remodeling studies, tendon constructs have been engineered in vitro using two techniques, self-organization of tenocytes around their autogenous collagen scaffold and tenocyte-induced contraction of fibrin gels. Functional bioreactors were designed and built for mechanical testing of constructs and also cyclic load intervention studies. Preliminary data have been collected that demonstrate cyclic mechanical loading increases collagen content and improves mechanical properties of the engineered constructs.

\subsection{Experimental Methods}

This section briefly describes the engineered construct methods. See Calve et al. (2004) for the detailed protocols for self-organized tendon constructs and Andrick et al. (2005) for more information on the formation of constructs by fibrin gel contraction.

\section{Self-organized construct formation}

Primary rat tendon fibroblasts were dissociated from Achilles tendons of Fischer 344 retired breeders and suspended in growth medium containing Ham F-12, 20\% fetal bovine serum (FBS) and 1\% antibiotic-antimycotic (Invitrogen 10437). The cells were expanded, passaged at approximately $60 \%$ confluence and stored in liquid nitrogen until needed. Preparation of 35-mm culture plates involved coating with $1.5 \mathrm{~mL}$ of silicone elastomer (SYLGARD), curing the SYLGARD for two weeks, rinsing, then adding natural mouse laminin in Dilbecco's phosphate-buffered saline (DPBS) and allowing the DPBS to evaporate. Two size 0 laminin-coated silk sutures were pinned $12 \mathrm{~mm}$ apart into the laminincoated substrate and the plates were filled with enough growth medium to cover the sutures then sterilized. The growth medium was aspirated and 200,000 cells suspended in $2 \mathrm{~mL}$ growth medium were seeded onto each plate and supplemented with ascorbic acid. Media were changed every 2-3 days until cells reached confluency after about 5 days. The medium was changed to differentiation medium (Dulbecco's Modified Eagle Medium containing $7 \%$ horse serum and $1 \%$ antibiotic-antimycotic) at 2-3 day intervals until constructs formed and were ready for mechanical intervention or testing. Construct formation occurred over a two-week period by the progressive delamination of the cell and autogenous collagen layer beginning at the outer periphery of the culture plate. The constructs formed into cylinders of length $12 \mathrm{~mm}$ and attached at their ends to the suture pins. The constructs remained viable for several weeks in culture under static tension due to cell contraction. A formed construct 3 months after plating is shown in Figure 2. 


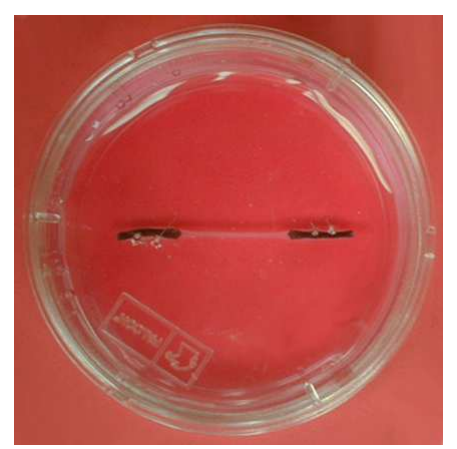

Figure 2. A 12-mm long self-organized engineered tendon construct in culture and suspended above the SYLGARD substrate via pinned silk sutures at its ends. The construct is shown at three months after cell plating [Calve et al. (2004)].

\section{Contracted fibrin gel formation}

Tenocytes were isolated from the Achilles tendon of Sprague Dawley rats using similar protocols as described above for the self-organized constructs. Culture plates were also prepared similarly; however, an oval (5 mm X $15 \mathrm{~mm}$ ) polydimethylsiloxane (PDMS) mold was pressed into the center of the plates prior to sterilization and cell plating. The growth medium was supplemented with $10 \mathrm{units} / \mathrm{mL}$ of thrombin and agitated. Fibrinogen was added and polymerized by the thrombin within 10 minutes. About 15 minutes after gel formation 100,000 tenocytes were seeded on the gel and the culture plates were incubated until ready for mechanical intervention or testing. The growth medium was changed after 2 days to differentiation medium to promote collagen deposition because constructs had completely formed around the oval molds.

\section{Histology}

Self-organized constructs were prepared for electron microscopy using histological methods described in Calve et al. (2004). Electron microscopy revealed the constructs contained $60 \mathrm{~nm}$ diameter collagen fibers oriented along the main axis of the construct, closely resembling the morphology of neonatal tendon. Histology methods for the fibrinbased constructs may be found in Andrick et al. (2005). Longitudinal sections stained with either Massons trichrome or picosirius red at three weeks after seeding showed that the cells have digested much of the fibrin and deposited and organized a collagen matrix.

\section{Mechanical testing}

Constructs formed by either approach were attached to functional bioreactors for cyclic mechanical loading. The constructs remained in culture and were attached to a stepper motor at one end and held fixed at the other end. The stepper motor was commissioned to cyclically strain the constructs between +10 and $-10 \%$ at $0.1 \mathrm{~Hz}$ for 7 days. The mechanical response curves of the constructs were determined using an optical device for in situ strain determination during tensile loading; details may be found in Arruda et al. (2005). Briefly, constructs were kept in a saline solution, their cross-sectional areas were determined by several diameter measurements, then they were attached to stepper 

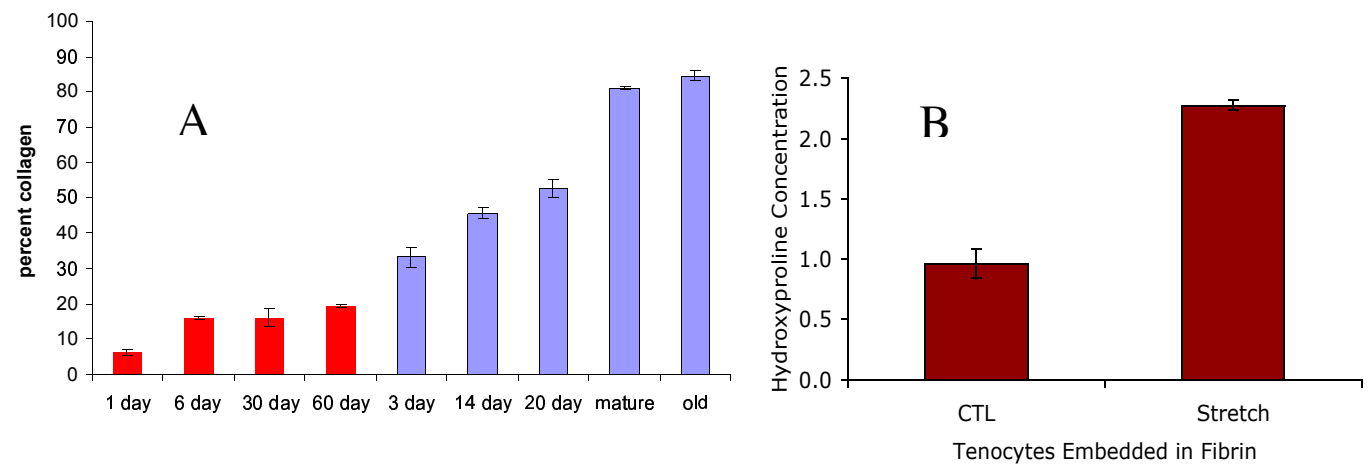

Figure 3. A Tenocytes in self-organized constructs are mechanoresponsive to static stretch. Collagen content vs. time in culture (first four bars) or animal age. B Tenocytes in fibrin-based constructs respond to cyclic stretching [Stretch] by depositing a greater than two-fold increase in collagen after one week of cyclic stretching compared to controls [CTL].

motors at either end, one end via an optical force transducer, and marked with India ink spots at several equal intervals along their lengths. Labview software controlled both servomotors as well as a digital video camera attached to a Nikon dissecting microscope. Constructs were loaded at a constrant strain rate, without preconditioning until failure and synchronized force and image recordings were collected and converted to nominal stress vs. nominal strain data.

\section{Collagen content determination}

The hydroxyproline assay of Woessner (1961) was used to determine collagen content in the constructs by assuming the hydroxyproline accounts for $13.8 \%$ of the total type I collagen.

\subsection{Experimental Results}

The tenocytes in the self-organized tendon constructs are mechanically responsive to static tensile stretch. Cells in control constructs held in bioreactors and in culture at the as-formed lengths respond to static tension by depositing collagen. Figure 3 demonstrates that the collagen content in the constructs increases over time as the construct grows. This response is similar to the growth response of tendon in vivo as an animal ages. The tenocytes in the engineered constructs also respond to cyclic stretching as shown in Figure 3. The collagen content increases as a result of cyclic stretching over that in the control constructs suggesting these constructs constitute a viable in vitro model for 
characterizing and determining evolution laws for collagen growth in soft tissue. Collagen growth is manifested in the stress-strain response curves of the tendon constructs as improvements in the mechanical properties associated with tendon function. Figure 4 demonstrates that cyclic stretching results in an evolution of the construct response curve towards that of native young tendon as the collagen content is increased, characterized by improved tangent stiffness and strength over those of controls held under static stretch for an equivalent amount of time.

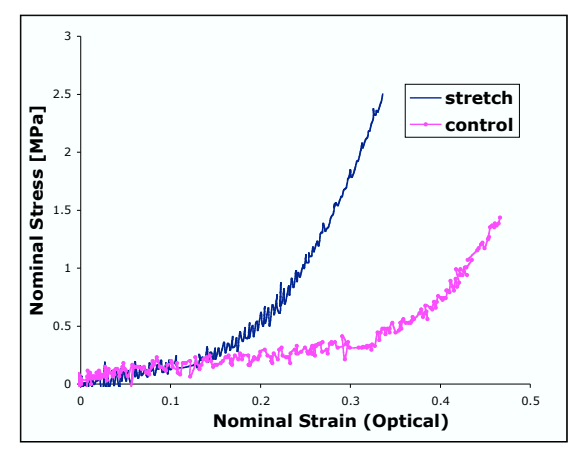

Figure 4. Stress-strain response curves of self-organized tendon constructs with (solid line) and without (line with symbols) cyclic stretching for approximately one week. The result of cyclic stretching includes an evolution of the mechanical properties of the construct, such as the shape of the initial toe region, tangent modulus and strength, towards those of young native tendon.

\section{Mathematical Modeling of Remodeling}

The mathematical model of remodeling in biology is treated as a motion in material space or configurational change. Further details of this remodeling framework may be found in Garikipati et al. (2005). The kinematics of remodeling are illustrated in Figure 5. The deformation gradient of the overall material motion is $\mathbf{F}$ and admits the multiplicative decomposition $\mathbf{F}=\mathbf{F}^{*} \mathbf{K}^{\mathbf{c}} \mathbf{K}^{\mathbf{r}}$, where $\mathbf{K}^{\mathbf{r}}$ is the tangent map to the preferred remodeled state that a region would attain if it were free to remodel according to the local cellular mechanotransductive responses. Evolution laws based on experimental results from, e.g., uniformly remodeling tissue would yield $\mathbf{K}^{r}$. It is incompatible in general because of constraints placed on the region by the surroundings and $\mathbf{K}^{c}$ represents the compatibility restoring tangent mapping to the remodeled configuration. The deformation gradient $\mathbf{F}^{*}$ maps to the current configuration.

\subsection{Outline of Remodeling Theory}

The mechanical theory may be examined by minimizing the following potential energy functional in the remodeled configuration $\boldsymbol{\Omega}_{t}^{*}$ and the total material motion $\kappa+\mathbf{u}^{*}$ :

$$
\boldsymbol{\Pi}\left[\mathbf{u}^{*}, \kappa\right]:=\int_{\boldsymbol{\Omega}_{\mathbf{t}}^{*}} \hat{\psi}^{*}\left(\mathbf{F}^{*}, \mathbf{K}^{\mathbf{c}}, \mathbf{X}^{*}\right) \mathrm{d} \mathbf{V}^{*}-\int_{\boldsymbol{\Omega}_{\mathbf{t}}^{*}} \mathbf{f}^{*} \cdot\left(\mathbf{u}^{*}+\kappa\right) \mathrm{d} \mathbf{V}^{*}-\int_{\partial \boldsymbol{\Omega}_{\mathbf{t}}^{*}} \overline{\mathbf{t}}^{*} \cdot\left(\mathbf{u}^{*}+\kappa\right) \mathrm{d} \mathbf{A}^{*}
$$




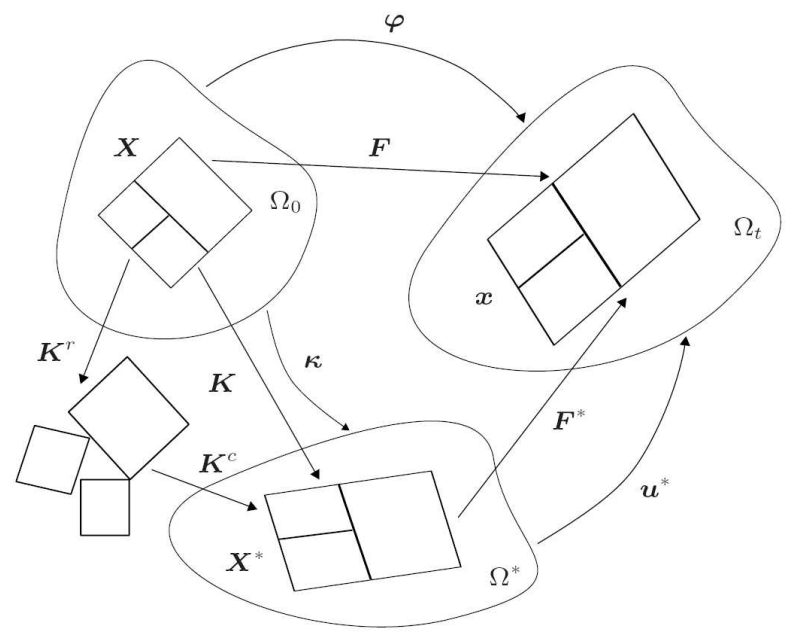

Figure 5. The kinematics of remodeling [Garikipati et al. (2005)].

where $\kappa$ is the motion of a point in material space (configurational change), $\mathbf{u}^{*}$ is the displacement, $\psi^{*}=\hat{\psi}^{*}\left(\mathbf{F}^{*}, \mathbf{K}^{\mathbf{c}}, \mathbf{X}^{*}\right)$ is the stored energy function and is assumed to depend on the compatibility restoring motion $\mathbf{K}^{c}$ in addition to $\mathbf{F}^{*}, \mathbf{f}^{*}$ is the body force and $\overline{\mathbf{t}}^{*}$ is the surface traction. Stationarity is assumed with respect to both displacements $\mathbf{u}^{*}$ and $\kappa$. Standard manipulations using variational calculus lead to the Euler-Lagrange equations that can be localized to the relations in $\boldsymbol{\Omega}^{*}$

$$
\begin{gathered}
\operatorname{Div}^{*} \mathbf{P}^{*}+\mathbf{f}^{*}=\mathbf{0} \\
-\operatorname{Div}\left[\psi^{*} \mathbf{I}-\mathbf{F}^{* \mathbf{T}} \mathbf{P}^{*}+\frac{\partial \psi^{*}}{\partial \mathbf{K}^{\mathbf{c}}} \mathbf{K}^{\mathbf{c} \mathbf{T}}\right]+\frac{\partial \psi^{*}}{\partial \mathbf{X}^{*}}=0,
\end{gathered}
$$

and on $\partial \boldsymbol{\Omega}^{*}$

$$
\begin{gathered}
\mathbf{P}^{*} \mathbf{N}^{*}=\overline{\mathbf{t}}^{*} \\
{\left[\psi^{*} \mathbf{I}-\mathbf{F}^{* \mathbf{T}} \mathbf{P}^{*}+\frac{\partial \psi^{*}}{\partial \mathbf{K}^{\mathbf{c}}} \mathbf{K}^{\mathbf{c} \mathbf{T}}\right] \mathbf{N}^{*}=\mathbf{0}}
\end{gathered}
$$

where $\mathbf{P}^{*}$ is the first Piola-Kirchhoff stress, $\psi^{*} \mathbf{I}-\mathbf{F}^{* \mathbf{T}} \mathbf{P}^{*}=\mathcal{E}$ is the Eshelby stress and $\frac{\partial \psi^{*}}{\partial \mathbf{K}^{\mathbf{c}}} \mathbf{K}^{\mathbf{c} \mathbf{T}}=\boldsymbol{\Sigma}$ is an additional configurational stress arising from the assumption that the configurational change stores energy. The remodeling is subjected to restrictions placed by the dissipation inequality for the mechanical theory, written in terms of the Kirchhoff stress

$$
\tau=\operatorname{det}[\mathbf{K}] \frac{\partial \psi^{*}}{\partial \mathbf{F}^{*}} \mathbf{F}^{* \mathbf{T}}
$$

as 


$$
\tau:\left(\dot{\mathbf{F}} \mathbf{F}^{-\mathbf{1}}\right)-\frac{\partial}{\partial \mathbf{t}}\left(\operatorname{det}[\mathbf{K}] \psi^{*}\right) \geq \mathbf{0},
$$

where $\mathbf{K}=\mathbf{K}^{\mathbf{c}} \mathbf{K}^{\mathbf{r}}$. This leads to the following reduced dissipation inequality, placing a restriction on the evolution law for $\mathbf{K}^{r}$ and on the functional form of the extra configurational stress $\boldsymbol{\Sigma}$ :

$$
-\operatorname{det}[\mathbf{K}](\mathcal{E}+\boldsymbol{\Sigma}):\left(\dot{\mathbf{K}}^{\mathbf{c}} \mathbf{K}^{\mathbf{c}-\mathbf{1}}\right)-\operatorname{det}[\mathbf{K}] \mathcal{E}:\left(\mathbf{K}^{\mathbf{c}} \dot{\mathbf{K}}^{\mathbf{r}} \mathbf{K}^{-\mathbf{1}}\right)-\operatorname{det}[\mathbf{K}] \frac{\partial \psi^{*}}{\partial \mathbf{X}^{*}} \geq \mathbf{0} .
$$

\subsection{A Remodeling Example}

The remodeling theory is illustrated using the anisotropic eight-chain model of Bischoff et al. Bischoff et al. (2002a,b) to describe a collagen network in a soft tissue such as tendon. Consider the case of a local tendon region initially described by the network in Figure $6 \mathbf{A}$, characterized by the fiber angle $\theta_{0}$ as shown. If the tendon were stretched uniaxially its true stress vs. true strain response curve would follow the dashed trace in Figure $6 \mathbf{C}$ as the collagen fibers orient via an affine transformation that is the tangent map of the incompressible deformation of the tissue. The network is hyperelastic, and in the absence of remodeling, it loads and unloads along the non-linear dashed path to return to the reference configuration of Figure $6 \mathbf{A}$ with the fibers returning to their original orientation as the deformation is removed. If instead the tendon region is described by a different fiber direction $\theta$ as shown in Figure $6 \mathbf{B}$, its response curve would follow the solid hyperelastic trace in Figure $6 \mathbf{C}$.

This remodeling example is simplified for purposes of illustration to assume the entire tissue is homogeneous, moreover it remodels homogeneously and therefore the remodeling is compatible. The kinematics illustrated in Figure 5 are simplified for this case to $\mathbf{K}^{\mathbf{c}}=\mathbf{I}$ which greatly simplifies the reduced dissipation inequality above. The first term is eliminated because $\dot{\mathbf{K}}^{\mathbf{c}}=\mathbf{0}$ and the third term is also zero because the strain energy density function is assumed uniform throughout the homogeneous tissue. The remaining term in the reduced dissipation inequality is also simplified in this case and the restriction placed on the remodeling evolution law, $\dot{\mathbf{K}}^{\mathbf{r}}$, is simplified by the requirement that the fibers in the network remodel towards the principal tensile stretch direction during uniaxial tension loading. We now consider remodeling during uniaxial loading of the network in Figure $6 \mathbf{A}$ such that the collagen fibers remodel towards the principal stretch direction in addition to deform in an affine manner during each loading step. The gradual remodeling evolves the reference configuration of the collagen fibers from that of Figure $6 \mathbf{A}$ to that of Figure $6 \mathbf{B}$ during the deformation to a true strain of 0.5 . At the end of this loading step the tissue is fixed in its evolved configuration and unloaded. The response of the remodeling tissue is shown by the trace with symbols in Figure $6 \mathbf{C}$. The simulation demonstrates that as the remodeling occurs during loading the network stores less strain energy at any given applied deformation level than it does if it deforms without remodeling. Remodeling occurs in this manner to relieve the excess strain energy of the applied deformation in much the same way tissue is hypothesized to remodel in response to increases in loading such as during exercise. The network is fixed in the remodeled configuration at the end of loading, and no further evolution of the microstructure occurs 

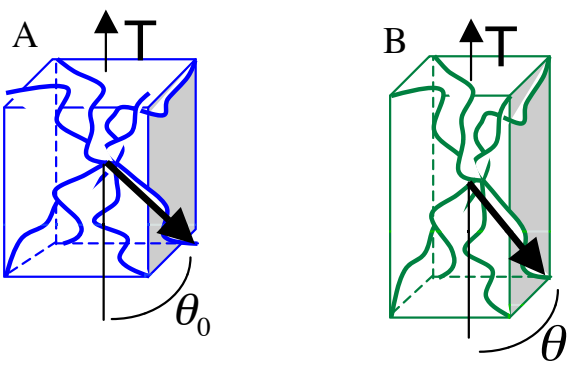

$\mathrm{C}$

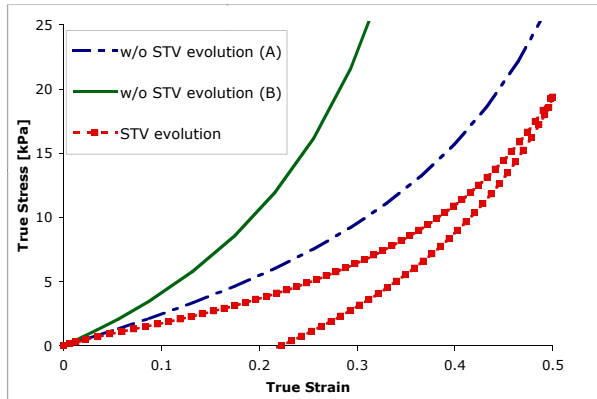

Figure 6. Numerical simulation of uniform remodeling of an anisotropic network during uniaxial deformation. A Hyperelastic network and original reference configuration of network that remodels during deformation, B Hyperelastic network and remodeled reference configuration of network that has remodeled during uniaxial loading, C Hyperelastic load-unload response curves for two distinct networks (i.e. (A), dashed curve; and (B), solid curve), and an additional response curve (with symbols) of a network that remodels from reference configuration (A) to remodeled reference configuration (B) during uniaxial loading and is fixed in remodeled configuration during unloading.

as the network is unloaded. The change in reference configuration is evidenced by the permanent deformation that remains upon fully unloading.

\section{Mathematical Modeling of Growth}

The continuum thermodynamics treatment of a system that is open with respect to mass to allow species concentration changes due to mass transport is now considered. The full details of the theory may be found in Garikipati et al. (2004). It is outlined here to demonstrate the differences between the growth and remodeling theories and to illustrate a simple growth example. The kinematical framework assumes local growth by $\mathbf{F}^{\mathbf{g}}$ resulting in an incompatible tissue. Elastic deformation by $\tilde{\mathbf{F}}^{\mathbf{e}}$ restores compatibility resulting in internal stresses. The applied elastic deformation is $\overline{\mathbf{F}}^{\mathbf{e}}$. The entire mapping admits the multiplicative decomposition $\overline{\mathbf{F}}^{\mathbf{e}} \tilde{\mathbf{F}}^{\mathbf{e}} \mathbf{F}^{\mathbf{g}}$. Multiple species are modeled in the continuum setting. The biological processes by which nutrients are transported and converted to living tissue are described mathematically by sources and sinks. Fluxes are introduced for each species diffusing through the solid phase. In the soft tissue application of tendon the solid phase is type I collagen. Mass balance for a species $i$ is given as $\frac{\partial \rho_{0}^{i}}{\partial t}=\boldsymbol{\Pi}^{\mathbf{i}}-\nabla \cdot \mathbf{M}^{\mathbf{i}}$, where $\rho_{0}$ is the concentration, $\boldsymbol{\Pi}$ is the source term (or sink term) and $\mathbf{M}$ is the mass flux vector of the species. The simplest illustrative example of the theory involves two species, the solid collagen phase and a fluid phase. The latter is assumed to carry nutrients, enzymes, proteases etc. involved in the synthesis and breakdown of the collagen phase but the two-species approximation assumes these reaction products do 
not diffuse relative to the fluid phase. Mass balance for the solid phase becomes $\frac{\partial \rho_{0}^{s}}{\partial t}=\mathbf{\Pi}^{\mathbf{s}}$ and for the fluid phase it is $\frac{\partial \rho_{0}^{f}}{\partial t}=-\nabla \cdot \mathbf{M}^{\mathbf{f}}$. Balance of linear and angular momenta and energy are considered for each species and momenta and energy transfer among species are allowed by introducing $\mathbf{q}^{\mathbf{i}}$, the force per unit mass exerted on species $i$ by the other species present, and an interaction energy term $\tilde{e}^{i}$ transferred to species $i$ by all other species per unit mass of $i$. The full development of these equations is rather lengthy and the reader is referred to Garikipati et al. (2004) for the details. The entropy production inequality holds for the system as a whole. It is combined with the energy equation and balance laws to derive the following reduced entropy inequality for growth processes

$$
\begin{aligned}
& \sum_{\mathbf{i}}\left(\rho_{\mathbf{0}}^{\mathbf{i}} \frac{\partial \mathrm{e}^{\mathrm{i}}}{\partial \mathbf{F}^{\mathbf{e}^{\mathbf{i}}}}-\mathbf{P}^{\mathbf{i}}\left(\mathbf{F}^{\mathbf{g} \mathbf{i}}\right)^{\mathbf{T}}\right): \dot{\mathbf{F}}^{\mathbf{e} \mathbf{i}}+\sum_{\mathbf{i}} \rho_{\mathbf{0}}^{\mathbf{i}}\left(\frac{\partial \mathrm{e}^{\mathrm{i}}}{\partial \eta^{\mathbf{i}}}-\theta\right) \frac{\partial \eta^{\mathbf{i}}}{\partial \mathbf{t}}+ \\
& \sum_{\mathbf{i}}\left(\rho_{\mathbf{0}}^{\mathbf{i}} \frac{\partial}{\partial \mathbf{t}}\left(\mathbf{V}+\mathbf{V}^{\mathbf{i}}\right)-\rho_{\mathbf{0}}^{\mathbf{i}} \mathbf{g}-\nabla \cdot \mathbf{P}^{\mathbf{i}}+\nabla\left(\mathbf{V}+\mathbf{V}^{\mathbf{i}}\right) \mathbf{M}^{\mathbf{i}}\right) \cdot \nabla \\
& +\sum_{\mathbf{i}}\left(\rho_{\mathbf{0}}^{\mathbf{i}} \mathbf{F}^{\mathbf{i}^{-\mathbf{T}}}\left(\nabla \mathrm{e}^{\mathrm{i}}-\theta \nabla \eta^{\mathbf{i}}\right)\right) \cdot \nabla+\sum_{\mathbf{i}} \boldsymbol{\Pi}^{\mathbf{i}}\left(\mathrm{e}^{\mathrm{i}}+\frac{\mathbf{1}}{\mathbf{2}}\left\|\mathbf{V}^{\mathbf{i}}\right\|\right)+ \\
& \frac{\nabla \theta \cdot \mathbf{Q}^{\mathbf{i}}}{\theta}+\sum_{\mathbf{i}} \rho_{\mathbf{0}}^{\mathbf{i}} \frac{\partial \mathrm{e}^{\mathrm{i}}}{\partial \rho_{\mathbf{0}}^{\mathbf{i}}} \frac{\partial \rho_{\mathbf{0}}^{\mathbf{i}}}{\partial \mathbf{t}}-\sum_{\mathbf{i}} \mathbf{P}^{\mathbf{i}}:\left(\nabla \mathbf{V}^{\mathbf{i}}+\mathbf{F}^{\mathbf{e} \mathbf{i}} \dot{\mathbf{F}}^{\mathbf{g} \mathbf{i}}\right) \leq \mathbf{0}
\end{aligned}
$$

where for species $i, \mathbf{P}^{\mathbf{i}}$ is the partial first Piola-Kirchhoff stress, $e^{i}$ is the internal energy per unit mass, $\eta^{i}$ is the entropy per unit mass, $\mathbf{V}^{\mathbf{i}}$ is the velocity relative to the solid phase velocity $\mathbf{V}$, and $\mathbf{Q}^{\mathbf{i}}$ is the partial heat flux vector. Also, $\mathbf{g}$ is the body force and $\theta$ is temperature. Constitutive assumptions of the form $e^{i}=\hat{e}^{i}\left(\mathbf{F}^{\mathbf{e} \mathbf{i}}, \eta^{i}, \rho_{0}^{i}\right)$ are made for the thermodynamic variables to obtain the following restrictions on the constitutive relations from the reduced entropy inequality: $\mathbf{P}^{\mathbf{i}} \mathbf{F}^{\mathbf{g}} \mathbf{T}=\rho_{\mathbf{0}}^{\mathbf{i}} \frac{\partial \mathbf{e}^{\mathbf{i}}}{\partial \mathbf{F}^{\mathbf{i}}}$, hyperelastic constitutive laws for the partial stresses; $\theta=\frac{\partial e^{i}}{\partial \eta^{i}} \rho_{0}^{i}$, temperature definition; $\mathbf{Q}^{\mathbf{i}}=-\mathbf{K}^{\mathbf{i}} \nabla \theta$, the Fourier Law of heat conduction; and a constitutive relation for the flux as a product of a mobility and a thermodynamic driving force in which the full coupling between mechanics and mass transport emerges,

$$
\mathbf{M}^{\mathbf{i}}=\mathbf{D}^{i} \cdot\left[\rho_{\mathbf{0}}^{\mathbf{i}} \mathbf{F}^{\mathbf{T}} \mathbf{g}+\mathbf{F}^{\mathbf{T}} \nabla \cdot \mathbf{P}^{\mathbf{i}}-\rho_{\mathbf{0}}^{\mathbf{i}}\left(\nabla \mathbf{e}^{\mathbf{i}}-\theta \nabla \eta^{\mathbf{i}}\right)\right]
$$

Specifically, mass transport is driven by an inertia term, a body force term (e.g. gravity), a stress divergence term such as pressure gradient and a chemical potential gradient. This last term includes strain gradient-dependent diffusion through the gradient in internal energy term and a concentration gradient-dependent diffusion arising from the mixing entropy. This theory has been implemented in the general purpose finite element program FEAP using the anisotropic eight-chain hyperelastic constitutive law of Bischoff et al. (2002a) for the solid collagen phase and a growth law for collagen that prescribes conversion to solid if the fluid concentration exceeds a preset value. A cylindrical construct of the size of the in vitro tendon construct models described earlier is simulated. It is assumed to initially contain a uniform solid volume fraction of 0.5 . The stress-strain 
response curve for this construct is first simulated without allowing growth and axially deforming it. The construct is then placed in a fluid bath and the collagen concentration is commissioned to increase locally if the fluid concentration in the construct exceeds its initial value of 0.5 . When placed in the fluid bath the construct swells as fluid flows from the high concentration bath to the low concentration construct. Solid is grown because of the rise in fluid concentration; this results in further swelling of the construct. The average final solid concentration is approximately 0.6 . After a period of growth the constitutive properties of the construct are again simulated by conducting a tensile test without allowing further growth. The results appear in Figure 7 as stress on the vertical axis and extension on the horizontal. They may be compared with the experimental results of growth in Figure 4 to show the theory is capable of capturing the non-linear constitutive response of the tendon constructs and correctly predicts the qualitative change in tangent modulus with collagen growth. However, further experiments of the type introduced here using in vitro soft tissue models are needed to provide accurate evolution laws for mathematical growth theories.

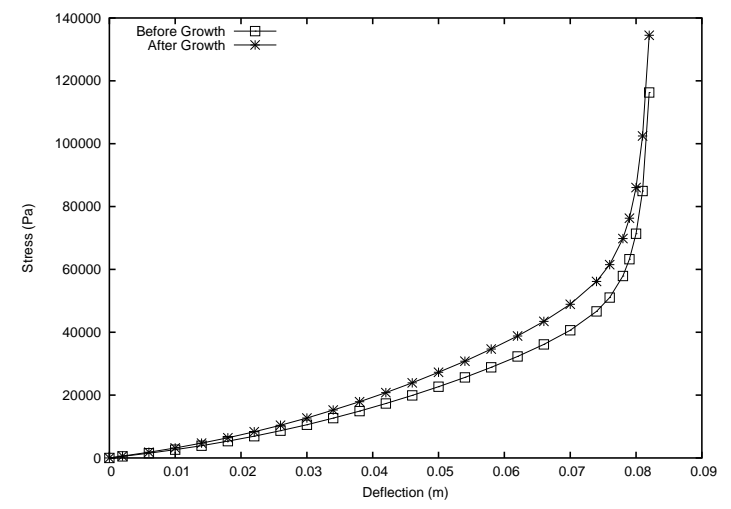

Figure 7. Results from a finite element simulation of growth in soft tissue. After an increase in collagen concentration from $50 \%$ (solid curve) to approximately $60 \%$ (dashed curve) the tangent stiffness of the construct is increased in agreement with experimental results.

\section{Conclusions}

Separate theories of growth and remodeling have been outline to illustrate that remodeling may occur at constant mass and is a configurational change whereas growth involves a change in the concentration of species. Engineered tendon constructs were generated for growth and remodeling studies. The constructs demonstrate mechanically responsive cells, grow and remain viable in culture for several weeks. They are excellent in vitro models for growth studies.

Acknowledgements This research was funded in part by DARPA (SPAWAR) contract N66001-02-C-8034, NSF (CMS) contract 9988693, and an LDRD sub-contract from Sandia National Laboratories. 


\section{References}

Andrick, J. J., and Mundy, K., and Calve, S. C., and Arruda, E. M., and Baar, K. (2005). Uniaxial stretch results in increased collagen in fibrin-based 3D engineered tendon. Submitted to J. Appl. Physiol.

Arruda, E. M., and Mundy, K., and Calve, S. C., and Baar, K. (2005). Denervation decreases tendon extensibility and increases tendon stiffness. Submitted to J. Physiol.

Bischoff, J. E., and Arruda, E. M., and Grosh, K. (2002). A microstructurally based orthotropic hyperelastic constitutive law. J. Appl. Mech. 69:570-579.

Bischoff, J. E., and Arruda, E. M., and Grosh, K. (2002). Orthotropic hyperelasticity in terms of an arbitrary molecular chain model. Tissue Eng. 10(5/6):755-761.

Calve, S. C., and Dennis, R. G., and Kosnik II, P. E., and Baar, K., and Arruda, E. M. (2004). Engineering of functional tendon. J. Appl. Mech. 69:199-201.

Epstein, M., and Maugin, G. A. (2000). Thermomechanics of volumetric growth in uniform bodies. Int. J. Plasticity 16:951-978.

Garikipati, K., and Arruda, E. M., and Grosh, K., and Narayanan, H., and Calve, S. C. (2004). A continuum treatment of growth in biological tissue: The coupling of mass transport and mechanics. J. Mech. Phys. Solids. 52:1595-1625.

Garikipati, K., and Narayanan, H., and Arruda, E. M., and Grosh, K., and Calve, S. C. (2005). Material forces in the context of biotissue remodelling. In Steinmann, P. and Maugin, G.A., eds., Mechanics of Material Forces. Dordrecht: Kluwer Academic Publishers. E-print available at http://arXiv.org/abs/q-bio.QM/0312002.

Humphrey, J. D., and Rajagopal, K. R. (2002). A constrained mixture model for growth and remodeling of soft tissue. Math. Models Meth. Appl. Sci. 12(3):407-430.

Klisch, S. M., and van Dyke, T. J. (2001). A theory of volumetric growth for compressible elastic biological materials. Math. Mech. Solids 6(6):551-575.

Kuhl, E., and Steinmann, P. (2002). Geometrically nonlinear functional adaptation of biological microstructures. In Mang, H., Rammerstorfer, F., Eberhardsteiner, J., eds., Proceedings of the Fifth World Congress on Computational Mechanics. International Association for Computational Mechanics. 1-21.

Nordin, M., and Lorenz, T., and Campello, M. (2001). Biomechanics of tendons and ligaments. In Nordin, M. and Frankel, V. H., eds., Basic Biomechanics of the Musculoskeletal System. New York: Lippincott Williams and Wilkins. 102-125.

Sengers, B. G., and Oomens, C. W. J., and Baaijens, F. P. T. (2004). An integrated finite-element approach to mechanics, transport and biosynthesis in tissue engineering. J. BioMech. Eng. 126(1):82-91.

Taber, L. A., and Humphrey, J. D. (2001). Stress-modulated growth, residual stress and vascular heterogeneity. J. BioMech. Eng. 123:528-535.

Woessner Jr., J. F. (1961). The determination of hydroxyproline in tissue and protein samples containing small proportions of this imino acid. Arch. Biochem. Biophys. 93:440-447. 